English

\title{
Health Questionnaire
}
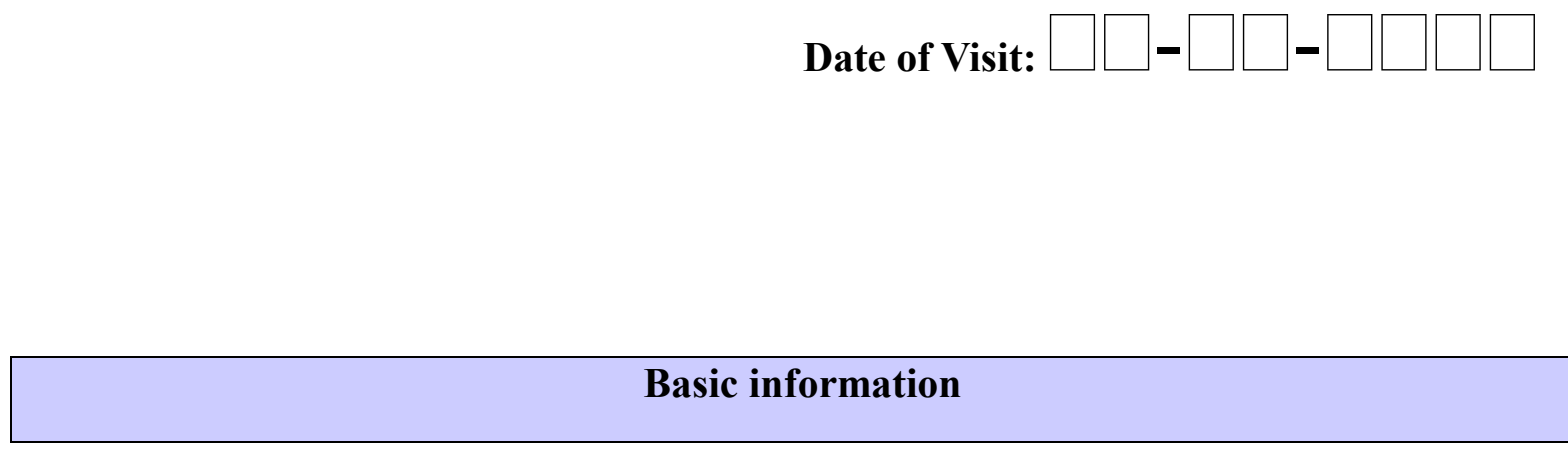

Name Last Name:

First Name:

Gender Male $\square \quad$ Female $\square$

Date of Birth (M-D-Y): $\square \square-\square \square-\square \square \square \square$ 


\section{Past Medical History}

$\square$ Not applicable to the below diagnosis

\begin{tabular}{|c|c|c|c|}
\hline Diagnosis & Yes & Medication & Remark \\
\hline Hypertension & $\square$ & $\square$ & \\
\hline Diabetes & $\square$ & $\square$ & \\
\hline Hyperlipidemia & $\square$ & $\square$ & \\
\hline $\begin{array}{c}\text { Angina/Myocardiac } \\
\text { Infarction }\end{array}$ & $\square$ & $\square$ & $\begin{array}{l}\square \text { Percutaneous coronary } \\
\text { intervention/Stent } \\
\square \text { Operation }\end{array}$ \\
\hline CVA (Stroke) & $\square$ & $\square$ & \\
\hline $\begin{array}{c}\text { Chronic Renal } \\
\text { Disease }\end{array}$ & $\square$ & $\square$ & $\begin{array}{l}\square \text { Decreased renal function } \\
\square \text { Dialysis }\end{array}$ \\
\hline Liver Cirrhosis & $\square$ & $\square$ & \\
\hline Chronic Hepatitis B & $\square$ & $\square$ & \\
\hline Chronic Hepatitis C & $\square$ & $\square$ & \\
\hline Others Diseases & $\square$ & & $\begin{array}{l}\square \text { Sites: } \\
\square \text { Diagnosis: }\end{array}$ \\
\hline
\end{tabular}

\begin{tabular}{|l|llll|}
\hline Cancer & $\square$ Lung & $\square$ Stomach & $\square$ Colon/Sigmoid & $\square$ Liver \\
& $\square$ Breast & $\square$ Cervix & $\square$ Thyroid & $\square$ Prostate \\
& $\square$ Other & & & \\
\hline
\end{tabular}

\begin{tabular}{|c|llc|}
\hline Abdominal & Lesion: & & \\
Surgery & $\square$ Stomach/Duodenum & $\square$ Colon/Sigmoid & $\square$ Appendix \\
& $\square$ Gall bladder & $\square$ Liver & $\square$ Pancreas \\
& $\square$ Kidney & $\square$ Uterus & $\square$ Ovary/Fallopian tube \\
& & & \\
& Diagnosis: & & \\
\hline
\end{tabular}

Other Surgery

Lesion:

Diagnosis: 


\section{Medicaion}

$\square$ Not applicable to the below medication

\begin{tabular}{|l|l|}
\hline \multicolumn{1}{|c|}{ Medication } & \multicolumn{1}{c|}{ Medication } \\
\hline$\square$ Aspirin & $\square$ Osteoporosis treatment \\
$\square$ Anti-coagulant (Warfarin/Coumadin) & $\square$ Calcium \\
$\square$ Anti-inflammatory agents & $\square$ Sedatives/Sleeping Pills \\
$\square$ Steroids & $\square$ Oriental Herbal Medication \\
$\square$ Thyroid Hormone & $\square$ Others: ( \\
$\square$ Antithyroid Agent & \\
&
\end{tabular}

\section{Eradication of Helicobacter pylori}

Have you ever had medications for eradication of Helicobacter pylori?

$\square$ No

$\square$ Yes $\quad \square \quad \square$ Successfully eradicated
$\square$ Failed to eradicate
$\square$ Do not know

\section{Family History}

Have any of your family members (parents, siblings or children) been diagnosed or died with any of the following diseases?

\begin{tabular}{|l|l|}
\hline \multicolumn{1}{|c|}{ Diagnosis } & \multicolumn{1}{c|}{ Diagnosis } \\
\hline$\square$ Hylertension & $\square$ Chronic hepatitis/Liver cirrhosis \\
$\square$ Diabdetes & $\square$ Liver Cancer \\
$\square$ Angina/Myocardiac Infarction (If & $\square$ Stomach Cancer \\
diagnosed under 55 years old in male or 65 & $\square$ Colon/Sigmoid Cancer \\
years old in female) & $\square$ Lung Cancer \\
$\square$ Cerebrovascular accident (Stroke) & \\
\hline
\end{tabular}




\section{Smoking History}

Have you smoked at least 5 packs (100 cigarettes) in the life?

$\square$ No

Smoked in the past, but quit

$\square$ How long has it been since you quit smoking cigarettes? ( ) year(s)

How long have you smoked? ( ) year(s)

How many cigarettes did you smoke a day? ( ) cigarette(s)

Currently smoking

How long have you been smoking? ( ) year(s)

How many cigarettes do you smoke a day? ( ) cigarette(s)

\section{Alcohol Drinking Habit}

How often do you drink alcohol?

- Per month: $\square$ less than once $\quad \square 2 \sim 4$ times

- Per week: $\quad \square$ twice $\quad \square 3$ times $\quad \square 4$ times $\quad \square 5$ times $\quad \square 6$ times $\quad \square 7$ times

How much do you usually drink at a time?

(Count each glass of the type of alcohol. Ex. Beer 1 can $(355 \mathrm{cc})=$ beer 1.6 glasses)

$\square$ 1 2 glass(es) $\quad \square 3 \sim 4$ glasses $\quad \square 5 \sim 6$ glasses

$\square$ 7 9 glasses $\quad \square$ more than 10 glasses 


\section{Exercise Related}

\begin{tabular}{|c|c|c|c|}
\hline $\begin{array}{l}\text { Think about all the vigorous activities (activities that } \\
\text { take hard physical effort and make you breathe much } \\
\text { harder than normal) that you did in the last } 7 \text { days. } \\
\text { Think only about those physical activities that you } \\
\text { did for at least } 10 \text { minutes at a time. During the last } 7 \\
\text { days, on how many days did you do vigorous } \\
\text { physical activities like heavy lifting, digging, } \\
\text { aerobics, or fast bicycling? }\end{array}$ & $\begin{array}{l}\square 0 \\
\square 2 \\
\square 4\end{array}$ & $\begin{array}{l}\square 1 \\
\square 3 \\
\square 5 \\
\square 7\end{array}$ & $\begin{array}{l}\text { How much time did } \\
\text { you usually spend } \\
\text { doing vigorous } \\
\text { physical activities } \\
\text { on one of those } \\
\text { days: } \\
\square \square \square \text { Minute(s)/day }\end{array}$ \\
\hline $\begin{array}{l}\text { Think about all the moderate activities (activities that } \\
\text { take moderate physical effort and make you breathe } \\
\text { somewhat harder than normal) that you did in the last } \\
7 \text { days. Think only about those physical activities that } \\
\text { you did for at least } 30 \text { minutes at a time. During the } \\
\text { last } 7 \text { days, on how many days did you do moderate } \\
\text { physical activities like carrying light loads, bicycling } \\
\text { at a regular pace, or doubles tennis? }\end{array}$ & $\begin{array}{l}\square 0 \\
\square 2 \\
\square 4\end{array}$ & $\begin{array}{l}\square 1 \\
\square 3 \\
\square 5 \\
\square 7\end{array}$ & $\begin{array}{l}\text { How much time did } \\
\text { you usually spend } \\
\text { doing moderate } \\
\text { physical activities } \\
\text { on one of those } \\
\text { days: } \\
\square \square \square \text { Minute(s)/day }\end{array}$ \\
\hline $\begin{array}{l}\text { Think about the time you spent walking in the last } 7 \\
\text { days. This includes at work and at home, walking to } \\
\text { travel from place to place, and any other walking that } \\
\text { you might do solely for recreation, sport, exercise, or } \\
\text { leisure. During the last } 7 \text { days, on how many days did } \\
\text { you walk for at least } 10 \text { minutes at a time? }\end{array}$ & $\begin{array}{l}\square 0 \\
\square 2 \\
\square 4\end{array}$ & $\begin{array}{l}\square 1 \\
\square 3 \\
\square 5 \\
\square 7\end{array}$ & $\begin{array}{l}\text { How much time did } \\
\text { you usually spend } \\
\text { walking on one of } \\
\text { those days: } \\
\square \square \square \text { Minute(s)/day }\end{array}$ \\
\hline
\end{tabular}

